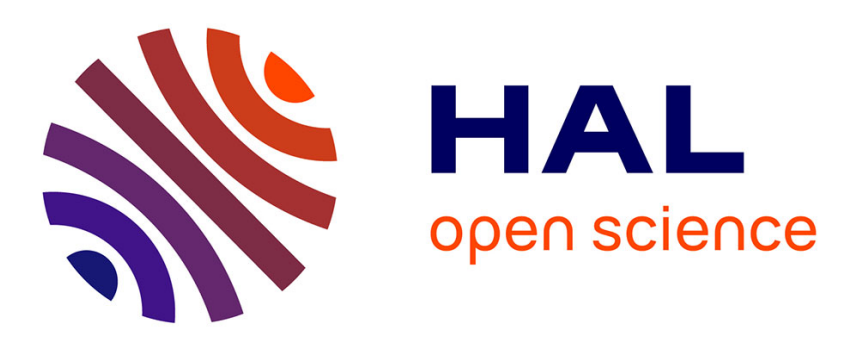

\title{
Gréation optique d'une inégalité de population entre les sous-niveaux Zeeman de l'état fondamental des atomes
}

Jean Brossel, Alfred Kastler, Jacques Winter

\section{To cite this version:}

Jean Brossel, Alfred Kastler, Jacques Winter. Gréation optique d'une inégalité de population entre les sous-niveaux Zeeman de l'état fondamental des atomes. J. Phys. Radium, 1952, 13 (12), pp.668-668. 10.1051/jphysrad:019520013012066800 . jpa-00234668

\section{HAL Id: jpa-00234668 https://hal.science/jpa-00234668}

Submitted on 1 Jan 1952

HAL is a multi-disciplinary open access archive for the deposit and dissemination of scientific research documents, whether they are published or not. The documents may come from teaching and research institutions in France or abroad, or from public or private research centers.
L'archive ouverte pluridisciplinaire HAL, est destinée au dépôt et à la diffusion de documents scientifiques de niveau recherche, publiés ou non, émanant des établissements d'enseignement et de recherche français ou étrangers, des laboratoires publics ou privés. 


\section{LETTRES A LA RÉDACTION}

\section{GREATION OPTIQUE D'UNE INÉGALITÉ DE POPULATION ENTRE LES SOUS-NIVEAUX ZEEMAN DE L'ETAT FONDAMENTAL DES ATOMES}

\author{
Par Jean Brossel, Alfred Kastien \\ et Jacques WinTER, \\ Laboratoire de Physique, \\ École Normale Supérieure.
}

L'un de nous [1] a suggéré un dispositif de " pompage optique ", permettant de créer une inégalité de population entre les sous-niveaux Zeeman de l'état fondamental des atomes.

Le cas envisagé était celui d'une transition $F_{1}=\frac{\mathrm{I}}{\text { ) }}$ à $F_{2}=\frac{\mathrm{I}}{2}$. Un champ magnétique sépare la raie en quatre composantes $\left(m_{F_{1}} \rightarrow m_{F_{0}}\right):\left(-\frac{\mathrm{I}}{2} \rightarrow-\frac{\mathrm{I}}{2}\right)$ et $\left(\frac{\mathrm{I}}{2} \rightarrow \frac{\mathrm{I}}{2}\right)$ ayant la polarisation $\pi,\left(-\frac{\mathrm{I}}{2} \rightarrow \frac{\mathrm{I}}{2}\right)$ ayant la polarisation circulaire droite $\sigma^{+}$et $\left(\frac{\mathrm{I}}{2} \rightarrow-\frac{\mathrm{I}}{2}\right)$ ayant la polarisation circulaire gauche $\sigma^{-}$.

Une excitation optique $\sigma_{1}^{+}$enlève des atomes à l'état fondamental $\left(-\frac{1}{2}\right)$ pour les porter à l'état excité $\left(+\frac{1}{2}\right)$. Lors de la réémission spontanée, deux raies sont émises : $\left(\frac{\mathrm{r}}{2} \rightarrow-\frac{\mathrm{I}}{2}\right)$ et $\left(\frac{\mathrm{I}}{2} \rightarrow \frac{\mathrm{I}}{2}\right)$. Si bien qu'une fraction, seulement des atomes excités revient au sous-niveau fondamental $\left(-\frac{1}{2}\right)$, le reste étant transféré en $\left(+\frac{I}{2}\right)$.

L'accumulation des atomes dans ce dernier état peut se poursuivre indéfiniment et l'on peut s'attendre à obtenir ainsi des proportions considérables d'atomes orientés.

L'expérience fut tentée sur la vapeur de mercure - pression de vapeur saturante à $0^{\circ} \mathrm{C}$ - par $\mathrm{J}$. Brossel et F. Bitter [2], et aucune orientation ne put être détectée.

Les collisions entre atomes et contre les parois sont des causes possibles de désorientation; mais, dans un jet atomique, leur importance est réduite au minimum.

L'expérience vient donc d'être reprise sur un jet atomique de sodium. Elle a donné un résultat positif, comme il est décrit ci-après.

Le jet était éclairé en $\sigma_{1}^{+}$sur $20 \mathrm{~cm}$ de long (la source lumineuse étant une lampe Philips SO 650). Le champ magnétique, d'une quinzaine de gauss, était perpendiculaire à la direction de propagation du jet et parallèle à la direction d'illumination $\sigma_{1}^{+}$.

Cette excitation préliminaire $\sigma_{1}^{+}$avait pour but de provoquer l'orientation des atomes, comme il est expliqué plus haut.

La détection de l'orientation s'effectuait de la manière suivante :

Après avoir passé la zone d'excitation $\sigma_{1}^{+}$, les atomes arrivaient dans une zone d'excitation $\pi$. Dans le cas où les sous-niveaux $-\frac{\mathrm{I}}{2}$ et $+\frac{\mathrm{I}}{2}$ de l'état fondamental ont même population, l'excitation crée un nombre égal d'atomes dans les sous-niveaux excités $-\frac{1}{2}$ et $+\frac{1}{2}$; et, lorsque les atomes retombent à l'état fondamental, les deux raies $\sigma_{2}^{+}$et $\sigma_{2}^{-}$apparaissent avec la même intensité. $\mathrm{Si}$, au contraire, les sous-niveaux $-\frac{\mathrm{I}}{2}$ et $+\frac{\mathrm{I}}{2}$ de l'état fondamental ont des populations inégales, l'excitation est suivie d'une réémission où les composantes $\sigma_{2}^{+}$et $\sigma_{2}^{-}$ont des intensités différentes.

L'expérience consiste à mesurer l'intensité $\sigma_{2}^{+}$ (ou $\sigma_{2}^{-}$) émise à la suite de l'excitation de détection $\pi$, en l'absence et en présence de l'excitation préalable $\sigma_{1}^{+}$. On trouve que l'excitation préalable $\sigma_{t}^{+}$ provoque une augmentation de $\sigma_{2}^{+}$et une diminution (égale) de $\sigma_{2}^{-}$, le rapport

$$
\frac{\Delta I_{\sigma^{+}}}{I_{\sigma_{+}}}=-\frac{\Delta I_{\sigma_{-}}}{I_{\sigma_{-}}}
$$

étant de l'ordre de i6 pour ioo.

L'existence du spin nucléaire du sodium ne change rien au principe de la méthode décrite plus haut. Dans les champs faibles utilisés, les effets observés correspondent à une inégalité de population entre les sous-niveaux $m_{F}$ de l'état fondamental. Des facteurs importants peuvent être gagnés sur $\Delta I_{\sigma_{+}}$ en utilisant des excitations $\sigma_{1}^{+}$plus intenses et sur de plus longs parcours. Il semble donc que le procédé décrit ici, permettra d'obtenir des taux d'orientation appréciables des atomes. Ces résultats sont à rapprocher de ceux obtenus par Rabi [3] en lumière totale.

$$
\text { Manuscrit reçu le } 14 \text { octobre } 1952 .
$$

[1] Kastler A. - J. Physique Rad., 1950, 11, 255; Physica, I 95 г, 17, г 9 г.

[2] Bitter F. et Brossel J. - Phys. Rev., 1952, 85, 1051.

[3] Rabi I. I. - Phys. Rev., i $952,87,379$. 